\title{
Microbial Population of Staphyloccous aureus from Inanimate Surfaces in a General Hospital Environment in Rivers State, Nigeria
}

\author{
AGBAGWA, OE; ONYEBULE, CQ
}

Department of microbiology faculty of Science University of Port Harcourt, Rivers state Nigeria. P.M.B. 5323 East- West Road Choba

Email: obakpororo.agbagwa@ uniport.edu.ng/ ejiroagbagwa@yahoo.com

\begin{abstract}
The isolation of Staphylococus aureus from inanimate objects in a General hospital in Rivers State was studied. Swabs were obtained from different sources comprising of; toilet seat, floor, switch, side rail bed, side rail chair, door handle, sink tap, bed spread, pillow case, and locker using the swab technique. High frequency of S. aureus was observed in toilet seat with perecentage distribution of $78 \%$, followed by floor with percentage distribution of $70 \%$ and locker with percentage distribution of $65 \%$ and the lowest frequency occurence was observed in switch with percentage distribution of $30 \%$. Frequency occurrence of S. aureus was $48.9 \%$ out of 50 isolates, indicating that antibiotics resistance pool especially betalactamase is to be widely available in the hospital environment and poor hygyine practices.
\end{abstract}

DOI: https://dx.doi.org/10.4314/jasem.v22i1.23

Copyright: Copyright (C) 2018 Agbagwa and Onyebule. This is an open access article distributed under the Creative Commons Attribution License (CCL), which permits unrestricted use, distribution, and reproduction in any medium, provided the original work is properly cited

Dates: Received 06 November 2017; received in revised form 3 December 2017; accepted 30 December 2017

Keywords: Inanimate surfaces, Staphyloccous aureus, Vancomycin -resistant ,

Staphpylococcus. aureus has the ability to resists various types of dry environments, including the surface of inanimate objects thought to be fomites. The hospital environment includes all surfaces outside a given patient zone, namely the healthcare facility environment and other patient zones. The Healthcare area may be contaminated by microorganisms from different patient zones (Sax et al., 2007). Hospital workers that come across patient in different zones of the hospital may cause crosstransmission and may further contaminate the environment due to poor hygiene practices (Pittet et al., 2006; Wertheim et al., 2005; Russotto et al., 2015). Methicillin-resistant $S$. aureus infection has become endemic in hospitals around the world despite conscious efforts by infection control professionals (Harbarths et al., 2003). Most MRSA are acquired in hospital and many of them can be prevented. MRSA can be transmitted between patients on the hands of the hospital staff, and hand washing is widely recognized as the single most important factor for prevention of colonization and infection (Boyce et al., 2003; Davis et al., 2012). Despite the implementation of standard infection control practices, the widespread failure to control $S$. aureus infection and MRSA is still on the increase. The hospital environment may be another important, but neglected factor in hospital cross-infection. Hospital Acquired MRSA strains are resistant to an array of antibiotics for which reason the treatment of infection due to this organisms is always a challenge, more so when the critically ill patients are affected. Contamination of the hospital inanimate environment by $S$. aureus and MRSA is a serious concern in human medicine which is a current trend in the world today. Historically hospital associated MRSA infections have predominated in humans and have contributed to significant illness and death (Simpson et al., 2007). Based on this there is a need to improve on the current situation, the necessity of mass screening of inanimate hospital environment for Methicillin Staphylococcus aureus must be evaluated by prospective studies, with the aim of determining the frequency of nosocomial MRSA in the hospital, and also to guide the development of local control and treatment policies. The study was carried out to ascertain the prevalence rate of $S$. aureus on inanimate surfaces within the hospital environment and the presence of resistant genes.

\section{MATERIAL AND METHODS}

Study area and sample collection: This study was carried out in five (5) different wards in a General hospital in Rivers State, Nigeria. Details on the source of sample are detailed in Table 1 below. Samples were collected using sterile swabs sticks (Bibby sterilin, Stone, Staffordshire, Uk) moistened with sterile saline and used to swab surface area of around $5 \times 5 \mathrm{~cm}$ of the various locations (Mizumachi et al. 2011) 
Isolation and Identification of Staphylococcus sp: Bacteria on the swabs were inoculated onto Mannitol Salt Agar (Oxoid) plates in an aseptic manner. All the inoculated plates were incubated under aerobic condition at $37^{\circ} \mathrm{C}$ for 24 hours to observe for developed colonies. A sterile wire loop was used to pick distinct colonies from Mannitol Salt Agar plates and streaked on Nutrient Agar plates. Plates were incubated at $37^{\circ} \mathrm{C}$ for 24 hours to observe for pure developed colonies of Staphylococcus aureus. The bacterial isolates were characterized and identified based on their colonial morphology and biochemical characteristics namely catalase test, coagulase test, fermentation, motility and Gram stain. Pure isolates of $S$. aureus were inoculated into slants and stored at $37^{\circ} \mathrm{C}$ for 24 hours (Cheesbrough, 2000).

Table .1: Source of Sample

\begin{tabular}{llllll}
\hline S/N & Isolate & Ward & S/N & Isolate & Ward \\
\hline 1 & TS & Female(fw) & 26 & DH & Accident \& emergency (A/E w) \\
2 & FL & Female(fw) & 27 & ST & Accident \& emergency (A/E w) \\
3 & Sw & Female(fw) & 28 & BS & Accident \& emergency (A/E w) \\
4 & SRB & Female(fw) & 29 & PC & Accident \& emergency (A/E w) \\
5 & SRC & Female(fw) & 30 & L & Accident \& emergency (A/E w) \\
6 & DH & Female(fw) & 31 & TS & Orthpaedic (O W) \\
7 & ST & Female(fw) & 32 & FL & Orthpaedic (O W) \\
8 & BS & Female(fw) & 33 & Sw & Orthpaedic (O W) \\
9 & PC & Female(fw) & 34 & SRB & Orthpaedic (O W) \\
10 & L & Female(fw) & 35 & SRC & Orthpaedic (O W) \\
11 & TS & Male(mw) & 36 & DH & Orthpaedic (O W) \\
12 & FL & Male(mw) & 37 & ST & Orthpaedic (O W) \\
13 & Sw & Male(mw) & 38 & BS & Orthpaedic (O W) \\
14 & SRB & Male(mw) & 39 & PC & Orthpaedic (O W) \\
15 & SRC & Male(mw) & 40 & L & Orthpaedic (O W) \\
16 & DH & Male(mw) & 41 & TS & Children emergency(CHEW) \\
17 & ST & Male(mw) & 42 & FL & Children emergency(CHEW) \\
18 & BS & Male(mw) & 43 & Sw & Children emergency(CHEW) \\
19 & PC & Male(mw) & 44 & SRB & Children emergency(CHEW) \\
20 & L & Accident \& emergency (A/E w) & 45 & SRC & Children emergency(CHEW) \\
21 & TS & Accident \& emergency (A/E w) & 46 & DH & Children emergency(CHEW) \\
22 & FL & Accident \& emergency (A/E w) & 47 & ST & Children emergency(CHEW) \\
23 & Sw & Accident \& emergency (A/E w) & 48 & BS & Children emergency(CHEW) \\
24 & SRB & Accident \& emergency (A/E w) & 49 & PC & Children emergency(CHEW) \\
25 & SRC & Accident \& emergency (A/E w) & 50 & L & Children emergency(CHEW)
\end{tabular}

Key: $\mathbf{T S}=$ Toilet seat $;$ SRC $=$ Side rail chair $\mathbf{F L ~}=$ Floor $; \mathbf{D H}=$ Door handle; $\mathbf{S W}=$ Switch $; \mathbf{S T}=$ Sink tap; $\mathbf{S R B}=$ Side rail bed;

BS $=$ Bed spread $; \mathbf{L}=$ Locker $; \mathbf{P C}=$ Pillow case

Antibiotic sensitivity test: The test was carried out using single disc-method. A loop-full of the isolated test organism was inoculated into saline solution to ensure the even distribution of the organism. This was then use to flood nutrient agar plate. Excess were discarded into disinfectant jar. Disc were aseptically placed on the agar plate and incubated at $37^{\circ} \mathrm{C}$ for 24 hours.

\section{RESULT AND DISCUSSION}

A total of fifty (50) surfaces from a General hospital in Rivers State Nigeria were screened for Staphylococcus aureus. The percentage distributions of isolates from different sources in female ward (fw) are shown in figure 1. Results shows that toilet seat $(16 \%)$ had the highest frequency next to floor (14\%) and door handles $(11 \%)$ while switch $(10 \%)$ had the lowest frequency of $S$. aureus. Percentage distribution of $S$. aureus from male ward (MW) is shown in Figure 2. Results shows that the floor (14\%) had the highest frequency of S. aureus next to toilet seat (10\%) and locker (11\%) while the bed spread (8\%) had the lowest frequency of $S$. aureus.

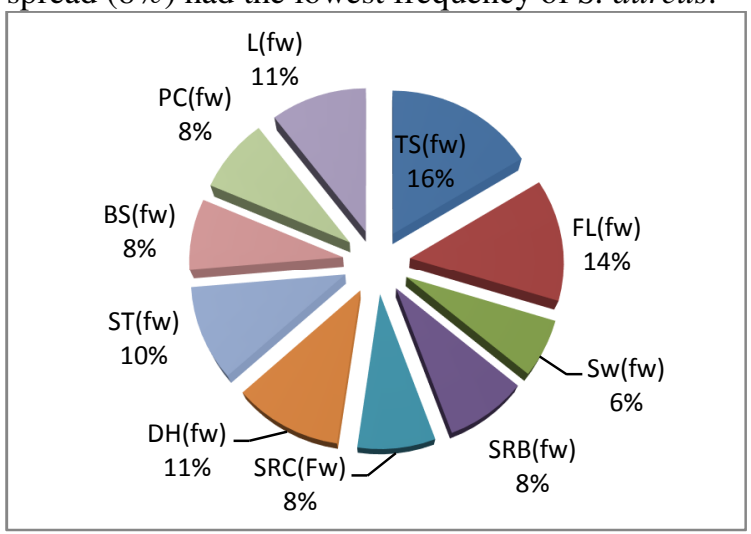

Fig 1: Percentage distribution of isolates from different sources in female Ward (fw)

Percentage distribution of $S$. aureus from children emergency ward (chew) is shown in Figure 3. Results shows that the side rail bed $(12 \%)$ had the highest frequency of S. aureus next to floor (12\%) and rail 
tap (11\%) while the pillow case (7\%) had the lowest frequency of $S$. aureus.



Fig 2: Percentage distribution of isolates from different sources in male ward (MW)

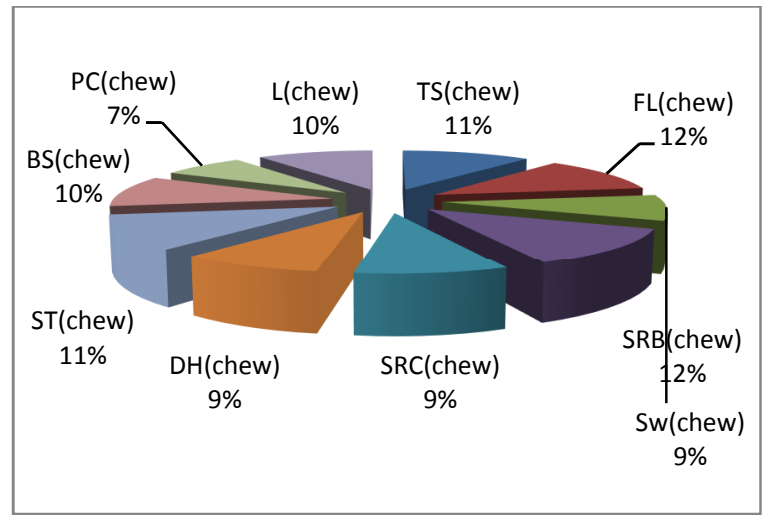

Fig 3: Distribution of Isolates from different sources in children emergency ward (Chew)

Percentage distribution of $S$. aureus from accident and emergency ward $(\mathrm{a} / \mathrm{e} \mathrm{w})$ is shown in figure 3.4. Result shows that the locker (10\%) had the highest frequency of $S$. aureus next to toilet seat (11\%) and door handle $(9 \%)$, while the side rail chair $(9 \%)$ had the lowest frequency of $S$. aureus. Percentage distribution of $S$. aureus from orthopedic ward (OW) is shown in Figure 3.5. Results shows that locker (13\%) had the highest frequency of $S$. aureus, the floor (10\%) and toilet seat (11\%), while the switch $(9 \%)$ and side rail chair had the lowest frequency of S. aureus $(8 \%)$.

The study was carried out to investigate the frequency of $S$. aureus in inanimate surfaces in hospital environment. Results obtained from the study revealed that the frequency of isolation of $S$. aureus in this in investigation was 50\%. Isolation of $S$. aureus from the inanimate areas of the hospital can be successfully accomplished with a combination of several microbiological analyses. The hospital environment represents a secondary reservoir of microorganisms such as Staph aureus (Nadia et al., 2016).

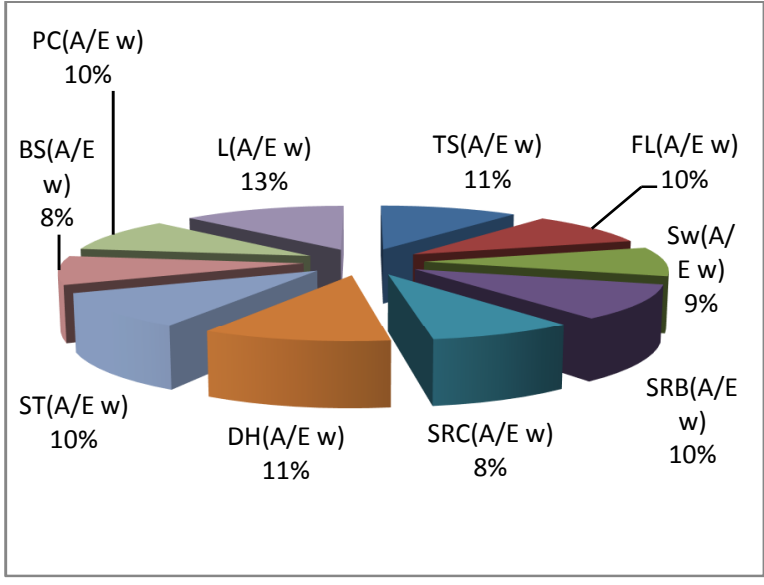

Fig 4: Distribution of isolates from different sources in accident and Emergency ward (a/e w)

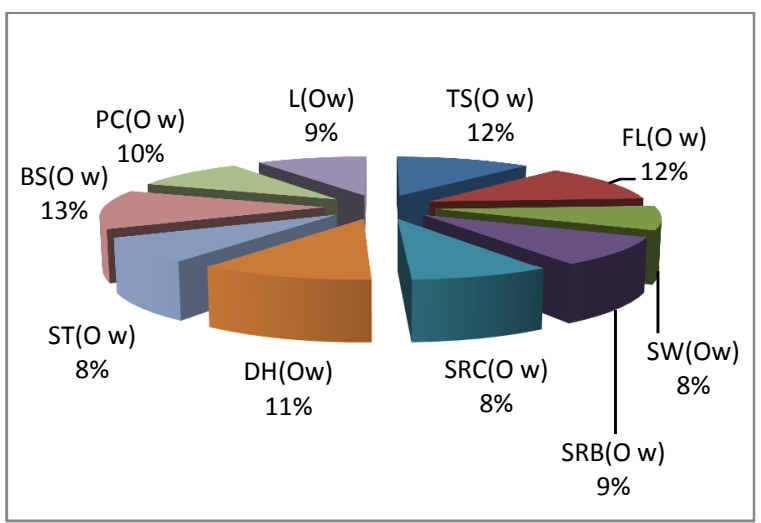

Fig 5: Distribution of isolates from different sources in orthopedic ward (OW)

They have the ability of colonizing almost any surface even fabrics (Neely and Maley, 2000; Wagenvoort et al., 2002; Chaibenjawog and Foster, 2011). The counts of $S$. aureus recorded in this study however may be related to the level of sanitation control policy in the health care unit where this study was conducted hence the urgent need for one. A study carried out in a hospital in Libya which revealed the highest count of MRSA in ICU and dialysis wards with percentage of $9.5 \%, 7.8 \%$ and $5 \%$ respectively (Nadia et al., 2016). The high frequency found in these inanimate objects is seen as a challenge because they have high frequency of contact with patient and are likely to transmit the disease. These underscores the necessity for a preventive intervention in our hospital, more importantly studies have shown that nosocomial spread of MRSA strains are harbored in inanimate environment of the hospital. Staphyloccoci can 
become pathogenic based on the toxins it produces at its stationary phase. These toxins produced by Staphyloccocus have the ability to destroy the host tissue thereby causing disease. Strains of Staphylococcus found in the hospital can cause different diseases and it is also responsible for nosocomial infections. High prevalence of Staphylococcus aureus in hospital settings can lead to MRSA associated diseases. A study carried out on emergency workers and bacterial contamination on touch surfaces showed that the carriage of MRSA was not affected by clinical exposure in the hospital because of the existing infection control policy in our hospital (Oguzkayaa- Artan et al., 2015).

Further advantage of eradication MRSA carried by hospital staff and institution of effective control policies in prevention of its transmission to healthy people visiting the hospital. In addition the financial implication of handling such hospital spread of MRSA is huge reason for which MRSA has been described as a public health issue with economic consequences (Mirelli et al., 2007). Resistance in commonly used antimicrobial such as cloxacillin has shown increasing prevalence in the last 15 years (Okeke et al., 2000). It is thought that sustained pressure like antibiotics may enhance the ability of microbes to resist the presence of antibiotics or drugs. Antibiotic resistance increasingly compromises the outcome of many infections that were until recently treatable and remains the most common diseases in Africa. The more often bacteria are exposed to antibiotics, the more likely that resistance will develop. The spread of infections that are resistant to antibiotic treatment has serious consequences for public health. It is advisable that prudent use of antibiotics and infection control, sanitation and hygiene practices are steps that must be taken now to stem the trend of rising resistance.

Conclusion: Staphylococcus isolated from the inanimate environment in the hospital showed various microbial load. The result obtained might be due to that the antibiotic gene pool especially beta lactamase that is responsible for a wide range of resistance in hospital environment. Thus there is need for the adoption, development and enforcement of appropriate control policies in hospitals with many points of critical care like our own where there are no existing or effective disease control programs, washing of hands and sterilization of hospital equipment.

\section{REFERENCES}

Boyce, JM; Potter-Bynoe, G; Chenevert, C; King, T (2003). Environmental contamination due to Methicillin-Resistant Staphylococcus aureus. 18:
$622-627$.

Chaibenjawong, P and Foster, SJ (2011). Desiccation tolerance in Staphylococcus aureus. Arch. Microbiol. 193:125-135.

Cheesbrough, M (2000). District laboratory practice in Tropical counties. Part 2. Cambridge University press, Cambridge. 62-70.

Davis, MF; Iverson, SA; Baron, P; Vasse, A; Silbergeld, EK; Lautenbach, E (2012). Household transmission of meticillinresistant Staphylococcus aureus and other staphylococci. Lancet Infect. Dis. 12:703-716.

Harbarth, S; Rutschmann, PS; Pittet, D (1998). Impact of Methicillin Resistance on the outcome of patients with bacteremia caused by Staphylococuss aureus. Arch. Intern. Med. 158: 182-189.

Mireille, GL; Pierre-Alexander, HD; Colin, V (2007). Methicillin Resistant Staphylococcus aureus: A public health issue with economic consequences. Can. S. Infect. Dis. Med. Microbial., 18:27-34.

Mizumachi, E; Kato, F; Hisatsune, J; Tsuruda, K; Uehara, Y; Seo, H (2011). Clonal distribution of enterotoxigenic Staphylococcus aureus on handles of handheld shopping baskets in supermarkets. J. Appl. Microbiol. 110:562-567.

Neely, AN; Maley, MP (2000). Survival of Enterococci and Staphylococci on hospital fabrics and plastic. J. Clin. Microbiol. 38:724726.

Okeke, IN; Fayinka, ST; Lamikanra, A (2000). Antibiotic resistance in Escherichia coli from Nigerian students, 1986 -1998. Emerg. Inf. Dis., 6(4): 393-396.

Oguzkaya-Artan, M; Baykan, Z; Artan, C; Avsarogullari, L (2015). Prevalence and risk factors for methicillin resistant Staphylococcus aureus carriage among emergency department workers and bacterial contamination on touch surfaces in Erciyes University Hospital, Kayseri, Turkey. African Health Sciences.15 (4):12891294. doi:10.4314/ahs.v15i4.31.

Pittet, D; Allegranzi, B; Sax, H; Dharan, S; PessoaSilva, CL; Donaldson, L (2006). Evidence-based model for hand transmission during patient care 
and the role of improved practices. Lancet Infect Dis, 6(10): 641-652.

Sampson, AHR; Dave, J; Cookson, B (2007). The value of routine screening of staff for Methicillin resistant Staphylococcus aureus in early onset ventilator associated Pneumonia. 10: 1-7.

Sax, H; Allegranzi, B; Uckay, I; Larson, E; Boyce, J; Pittet, D (2007). 'My five moments for hand hygiene': a user-centered design approach to understand, train, monitor and report hand hygiene. J Hosp Infect. 67(1):9-21.

Russotto, V; Cortegiani, A; Raineri, S M; Giarratano, A (2015). Bacterial contamination of inanimate surfaces and equipment in the intensive care unit. Journal of Intensive Care, 3, 54. http://doi.org/10.1186/s40560-015-0120-5
Wagenvoort, JH; Sluijsmans, W; Penders,

RJ( 2000). Better environmental survival of outbreak vs. sporadic MRSA isolates. J. Hosp. Infect. 45:231-234.

Wertheim, HF; Melles, DC; Vos, MC; van Leeuwen, W; van Belkum, A; Verbrugh, HA (2005).The role of nasal carriage in Staphylococcus aureus infections. Lancet Infect Dis. (12):751-762. 